\title{
Possible sertraline-induced extrapyramidal adverse effects in an adolescent
}

\author{
This article was published in the following Dove Press journal: \\ Neuropsychiatric Disease and Treatment \\ 6 May 2016 \\ Number of times this article has been viewed
}

\author{
Lian-fang Wang' \\ Jin-wen Huang' \\ Si-yang Shan ${ }^{2}$ \\ Jia-hong Ding ${ }^{3}$ \\ Jian-bo Lai ${ }^{1,2}$ \\ Yi $\mathrm{Xu}^{1,4}$ \\ Shao-hua $\mathrm{Hu}^{1,4}$ \\ 'Department of Psychiatry, The First \\ Affiliated Hospital, Zhejiang University \\ School of Medicine, ${ }^{2}$ Faculty of \\ Clinical Medicine, Zhejiang University \\ School of Medicine, Hangzhou, \\ Zhejiang, People's Republic of China; \\ ${ }^{3}$ Department of Psychology, University \\ of Hong Kong, Pokfulam, Hong Kong; \\ ${ }^{4}$ The Key Laboratory of Mental \\ Disorder's Management in Zhejiang \\ Province, National Clinical Research \\ Center for Mental Health Disorders, \\ Hangzhou, Zhejiang, People's Republic \\ of China
}

Correspondence: Shao-hua Hu Department of Psychiatry, The First Affiliated Hospital, Zhejiang University School of Medicine, 79 Qingchun Road, Hangzhou 310003, People's Republic of China

Tel $+8657 \mid 56723002$

$\mathrm{Fax}+8657 \mid 5672300$ ।

Email dorhushaohua@zju.edu.cn

\begin{abstract}
Sertraline has been considered to be a relatively safe selective serotonin reuptake inhibitor for adolescents for a long time. We report herein a case of a 16-year-old Chinese boy with depression who experienced extrapyramidal-like effects, for example, facial spasm, upper limb dystonia, akathisia, and other disturbed behaviors, while being treated with sertraline $200 \mathrm{mg}$ per day. His movement symptoms were significantly alleviated after the discontinuation of sertraline and the administration of scopolamine. This finding indicates that albeit infrequent, sertraline may cause severe extrapyramidal symptoms in adolescent patients, suggesting that clinicians should be alert to the neurological side effects of sertraline in young patients.
\end{abstract}

Keywords: adolescents, selective serotonin reuptake inhibitors, sertraline, extrapyramidal symptoms

\section{Introduction}

Sertraline, a selective serotonin reuptake inhibitor (SSRI), has long been used in major psychiatric disorders, especially in depression. Compared to other SSRI antidepressants, such as paroxetine and fluoxetine, sertraline is less often reported to induce treatment-emergent extrapyramidal symptoms (EPSs). ${ }^{1}$ According to the pharmacoepidemiological data revealed by the US Food and Drug Administration, approximately $10 \%$ of all SSRI-associated EPSs have a link to sertraline, which was documented exclusively in adult patients. ${ }^{2}$ However, the present case wherein sertraline-associated EPSs were found in an adolescent depressive patient helps to extend our knowledge.

\section{Case report}

A 16-year-old male student was admitted to the psychiatric inpatient unit because of depression. Albeit without any pertinent triggering events, he had developed depressed mood, tearfulness, helpless feelings, and sleeping disturbance 2 years before admission; and his symptoms worsened 1.5 years prior to admission. The patient failed to maintain attention and concentration, which presumably contributed to his poor academic performance. He gradually entertained suicidal thoughts. Disturbed behaviors were also observed, for example, scratching his head and crying, prior to hospitalization. In regard to his medical history, no developmental problems, intoxication, substance abuse, or pertinent neuropsychiatric conditions were mentioned. Additionally, no family history of any psychotic disorder was reported. After his admission, physical and hematological examinations were performed for the patient, as well as cranial structural magnetic resonance imaging and electroencephalography. However, no significant findings were reported. The patient was then provisionally diagnosed with major depressive disorder 
according to the Diagnostic and Statistical Manual of Mental Disorders, 4th Edition, Text Revision. Accordingly, the patient was treated with sertraline at an initial dose of $50 \mathrm{mg}$ per day. Meanwhile, sodium valproate (500 mg per day) was used as an adjunct therapy to stabilize his mood. When the dose of sertraline reached a maximum of $200 \mathrm{mg}$ per day, some mild gastrointestinal adverse effects were reported, including nausea and upset stomach. Aluminum magnesium carbonate was thereof prescribed to manage these side effects.

On the 20th day of sertraline treatment, the patient exhibited restlessness, irritation, and disturbed behaviors, for which alprazolam $(0.4 \mathrm{mg})$ was temporally used. The dosage of sertraline was also reduced. However, these symptoms did not alleviate. The situation worsened on the next day, and the noted abnormal behaviors escalated, manifesting as unprovoked grinning and paw-like gesture with fingers curled inward. Meanwhile, this patient could not stop stretching his neck forward. An instant physical examination revealed that his arm muscles were excessively tight. Immediate treatment with diazepam $(5 \mathrm{mg}$ ) did not relieve these symptoms. Subsequently, intramuscular injection with clonazepam (1 mg) was given. Besides, sertraline was directly discontinued. Real-time hematological tests revealed an elevated serum level of creatine phosphokinase at $692 \mathrm{mmol} / \mathrm{L}$.

On the third day since his in-hospital sufferings, the patient still experienced facial spasm, tight arms, and fingers curled inward. He was unable to stand or sit still, whereas his mood was stable. Considering his movement symptoms as extrapyramidal effects, intramuscular administration with scopolamine $(0.3 \mathrm{mg})$ twice per day was medicated, which was reduced to once per day in the following 2 days and discontinued after full remission in movement symptoms. Given his depressive symptoms remained unresolved, antidepressive treatment with citalopram was initiated in this patient and gradually escalated to $40 \mathrm{mg}$ per day. After a 2-week citalopram treatment, his depressive symptoms were significantly improved. In outpatient follow-ups, this patient continued to take citalopram and no secondary movement problems were observed. Of note, throughout the SSRI treatment, sodium valproate was maintained in the initial dosage.

This work was approved by the Institute Ethical Committee of The First Affiliated Hospital, Zhejiang University School of Medicine, and written informed consent was given by the patient and his guardian.

\section{Discussion}

In this present case, our patient's sufferings were markedly alleviated by scopolamine, and his movement manifestations were then attributed to sertraline-related EPSs. However, there are still several factors that seem to confound this conclusion. As was described in a literature review, SSRIs treatment with other concomitant medications may contribute to an increased risk of EPSs. ${ }^{2}$ In a total of 289 cases of reported EPSs, nine were correlated to the monotherapy of sertraline, whereas the remaining were ascribed to sertraline with concurrent psychotropics. ${ }^{2}$ The common types of EPSs associated with sertraline are tremor, akathisia, and dystonia, and the reported dose ranges from 50 to $150 \mathrm{mg}$ per day. Of note, these patients are all adults, with age range from 18 to 84 years. $^{2-4}$ In our case, the concomitant medication was sodium valproate, and, to our knowledge, very uncommon case reports have corroborated their association with EPSs. In patients being treated with sodium valproate, treatment-emergent EPSs are more likely to occur in older patients with physical illnesses, such as epilepsy, usually present as parkinsonism and are reversible after discontinuation. ${ }^{5-7}$ Meanwhile, sodium valproate was used throughout the SSRI treatment. Despite the fact that the use of sertraline in combination with sodium valproate did not allow for causality to be established, it must be noted that the EPSs in this patient were not alleviated until sertraline was discontinued and scopolamine was injected.

Another factor is the patient's preexisting medical conditions. Given the fact that our patient had been bitten by a stray dog 2 years before admission and his physical symptoms resembled those observed in patients with rabies, it was possible that he had contracted rabies. However, it is reported that the latency of rabies commonly ranges from 20 days to 2 months, and 6 years at most. ${ }^{8}$ With a negative serologic test for rabies antibodies, this possibility was later excluded. Based on the aforementioned evidence, we conclude that the manifestation of EPSs in our patient was associated with the exposure to sertraline.

The mechanism via which SSRIs induce EPSs has not yet been fully clarified. It has been suggested that SSRIs block the reuptake of serotonin, which may be accompanied with inhibitory effects on dopaminergic pathways in the midbrain as well as the forebrain. ${ }^{9}$ As for sertraline, the mild effect of dopaminergic inhibition in the ventral tegmental area and nigrostriatal tract is considered to be associated with the development of EPSs. ${ }^{2,10}$ From the perspective of molecular genetics, SSRI-induced EPSs may be also related to serotonin or dopamine receptor polymorphisms or cytochrome P450 phenotypes. ${ }^{11}$ In young patients, the incidence of SSRIs-associated EPSs lacks definitive clinical evidence, and possibly depends on specific pharmacokinetics, individual differences, and immature development of central nervous system. 


\section{Conclusion}

We are the first to report a potential association between sertraline and EPSs in an adolescent patient. Albeit infrequent, clinicians should be alert to the possibility of sertralineinduced EPSs, especially in children and adolescents. This single case report presents detailed and unique data for the feasibility of sertraline treatment in adolescents. Future clinical trials with larger samples may delve more convincingly into this issue.

\section{Acknowledgments}

This work was supported by a grant from the National Clinical Research Center for Mental Health Disorders (2015BAI13B02). The authors also acknowledge the patient and his guardian for their understanding.

\section{Disclosure}

The authors report no conflicts of interest in this work.

\section{References}

1. Haddad PM, Dursun SM. Neurological complications of psychiatric drugs: clinical features and management. Hum Psychopharmacol. 2008; 23 Suppl 1:15-26.

2. Madhusoodanan S, Alexeenko L, Sanders R, Brenner R. Extrapyramidal symptoms associated with antidepressants - a review of the literature and an analysis of spontaneous reports. Ann Clin Psychiatry. 2010;22: $148-156$.
3. Kölling P, Jansen Steur EN, de Burlet HM. Extrapyramidal disorders following sertraline use; experiences with a new selective antidepressant. Ned Tijdschr Geneeskd. 1996;140(28):1467-1468.

4. Lambert MT, Trutia C, Petty F. Extrapyramidal adverse effects associated with sertraline. Prog Neuropsychopharmacol Biol Psychiatry. 1998;22(5):741-748.

5. Masmoudi K, Gras-Champel V, Bonnet I, et al. Dementia and extrapyramidal problems caused by long-term valproic acid. Therapie. 2000; 55(5):629-634.

6. Schreur L, Middeljans-Tijssen CW, Hengstman GJ, Olde Rikkert MG. Cognitive impairment and parkinsonism due to use of sodium valproate. Tijdschr Gerontol Geriatr. 2009;40(1):29-33.

7. Ristić AJ, Vojvodić N, Janković S, Sindelić A, Sokić D. The frequency of reversible parkinsonism and cognitive decline associated with valproate treatment: a study of 364 patients with different types of epilepsy. Epilepsia. 2006;47(12):2183-2185.

8. Woldehiwet Z. Rabies: recent developments. Res Vet Sci. 2002;73: $17-25$.

9. Kapur S, Remington G. Serotonin-dopamine interaction and its relevance to schizophrenia. Am J Psychiatry. 1996;153:466-476.

10. Gill HS, DeVane CL, Risch SC. Extrapyramidal symptoms associated with cyclic antidepressant treatment: a review of the literature and consolidating hypotheses. J Clin Psychopharmacol. 1997;17:377-389.

11. Hedenmalm K, Güzey C, Dahl ML, Yue QY, Spigset O. Risk factors for extrapyramidal symptoms during treatment with selective serotonin reuptake inhibitors, including cytochrome $\mathrm{P}-450$ enzyme, and serotonin and dopamine transporter and receptor polymorphisms. J Clin Psychopharmacol. 2006;26:192-197.
Neuropsychiatric Disease and Treatment

\section{Publish your work in this journal}

Neuropsychiatric Disease and Treatment is an international, peerreviewed journal of clinical therapeutics and pharmacology focusing on concise rapid reporting of clinical or pre-clinical studies on a range of neuropsychiatric and neurological disorders. This journal is indexed on PubMed Central, the 'PsycINFO' database and CAS,

\section{Dovepress}

and is the official journal of The International Neuropsychiatric Association (INA). The manuscript management system is completely online and includes a very quick and fair peer-review system, which is all easy to use. Visit http://www.dovepress.com/testimonials.php to read real quotes from published authors. 